\title{
Uterine Ligament Serous Adenocarcinoma
}

National Cancer Institute

\section{Source}

National Cancer Institute. Uterine Ligament Serous Adenocarcinoma. NCI Thesaurus.

Code C40136.

A rare serous adenocarcinoma that arises from the uterine lig ament. 